\title{
MACHINE LEARNING-AIDED, ROBUST WIDEBAND SPECTRUM SENSING FOR COGNITIVE RADIOS
}

Sudharman Jayaweera

Department of Electrical and Computer Engineering University of New Mexico

Albuquerque, NM 87131

12 Jun 2015

Final Report

APPROVED FOR PUBLIC RELEASE; DISTRIBUTION IS UNLIMITED.

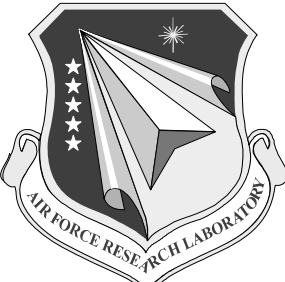

AIR FORCE RESEARCH LABORATORY Space Vehicles Directorate 3550 Aberdeen Ave SE AIR FORCE MATERIEL COMMAND KIRTLAND AIR FORCE BASE, NM 87117-5776 


\section{DTIC COPY NOTICE AND SIGNATURE PAGE}

Using Government drawings, specifications, or other data included in this document for any purpose other than Government procurement does not in any way obligate the U.S. Government. The fact that the Government formulated or supplied the drawings, specifications, or other data does not license the holder or any other person or corporation; or convey any rights or permission to manufacture, use, or sell any patented invention that may relate to them.

This report is the result of contracted fundamental research deemed exempt from public affairs security and policy review in accordance with SAF/AQR memorandum dated 10 Dec 08 and AFRL/CA policy clarification memorandum dated 16 Jan 09. This report is available to the general public, including foreign nationals. Copies may be obtained from the Defense Technical Information Center (DTIC) (http://www.dtic.mil).

\section{AFRL-RV-PS-TR-2015-0113 HAS BEEN REVIEWED AND IS APPROVED FOR PUBLICATION IN ACCORDANCE WITH ASSIGNED DISTRIBUTION STATEMENT.}

//SIGNED//

STEVEN A. LANE

Program Manager
//SIGNED//

PAUL HAUSGEN, Ph.D.

Technical Advisor, Space Based Advanced Sensing and Protection

//SIGNED//

JOHN BEAUCHEMIN

Chief Engineer, Spacecraft Technology Division

Space Vehicles Directorate

This report is published in the interest of scientific and technical information exchange, and its publication does not constitute the Government's approval or disapproval of its ideas or findings. 


\section{REPORT DOCUMENTATION PAGE}

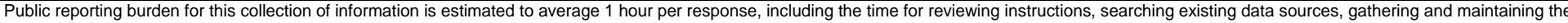

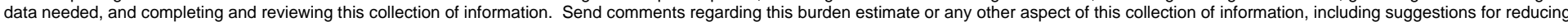

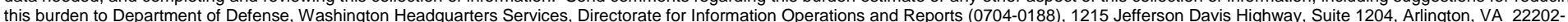

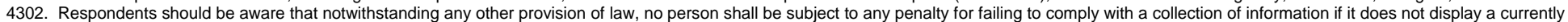
valid OMB control number. PLEASE DO NOT RETURN YOUR FORM TO THE ABOVE ADDRESS.
$12-06-2015$
2. REPORT TYPE
Final Report

4. TITLE AND SUBTITLE

Machine Learning-Aided, Robust Wideband Spectrum Sensing for Cognitive Radios

\section{AUTHOR(S)}

Sudharman Jayaweera
3. DATES COVERED (From - To) 29 Apr 2014 to 12 Jun 2015 5a. CONTRACT NUMBER

FA9453-14-1-0231

5b. GRANT NUMBER

5c. PROGRAM ELEMENT NUMBER $61102 \mathrm{~F}$

5d. PROJECT NUMBER 3001

5e. TASK NUMBER

PPM०००19614

5f. WORK UNIT NUMBER

EF122381

8. PERFORMING ORGANIZATION REPORT NUMBER

Department of Electrical and Computer Engineering University of New Mexico

Albuquerque, NM 87131

\section{SPONSORING I MONITORING AGENCY NAME(S) AND ADDRESS(ES)}

Air Force Research Laboratory

Space Vehicles Directorate

3550 Aberdeen Ave SE

Kirtland AFB, NM 87117-5776

12. DISTRIBUTION / AVAILABILITY STATEMENT

Approved for public release; distribution is unlimited.
10. SPONSOR/MONITOR'S ACRONYM(S)

AFRL/RVSV

11. SPONSOR/MONITOR'S REPORT NUMBER(S)

AFRL - RV - PS - TR - 2015- 0113

\section{SUPPLEMENTARY NOTES}

\section{ABSTRACT}

In this project, a compressive-sampling based, robust spectrum sensing approach was developed for wideband cognitive radios. The compressive-sampling based front-end is intended for overcoming the hardware imposed limitations on wideband spectrum sensing while robust detection principles are used to obtain a spectrum sensing approach that helps alleviate sensitivity to non-Gaussian noise and interference. Simulations were carried out to demonstrate that even with reduced number of samples, the proposed compressive-sampling based robust detector can indeed provide either comparable or better results to that observed with conventional periodogram, but with significantly higher number of samples. These results encourage further investigations and improvements of the proposed approach as a viable candidate for the front-end processing of a wideband cognitive radio.

15. SUBJECT TERMS

Space communication; satellite communication; digital signal processing; spectrum sensing

16. SECURITY CLASSIFICATION OF:

a. REPORT

Unclassified

\section{b. ABSTRACT}

Unclassified c. THIS PAGE

Unclassified
17. LIMITATION OF ABSTRACT

Unlimited

18. NUMBER
OF PAGES
22

19a. NAME OF RESPONSIBLE PERSON
Steven A. Lane
19b. TELEPHONE NUMBER (include
area code)


(This page intentionally left blank)

Approved for public release; distribution is unlimited. 


\section{TABLE OF CONTENTS}

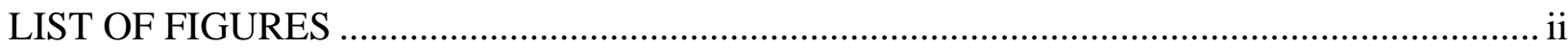

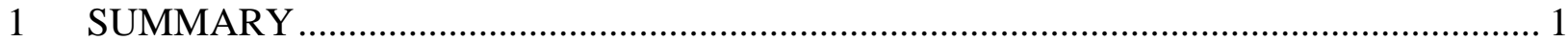

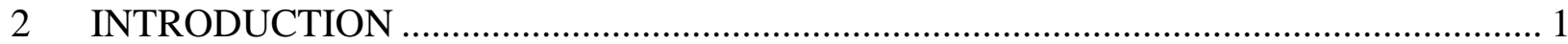

3 METHODS, ASSUMPTIONS, AND PROCEDURES ..................................................... 3

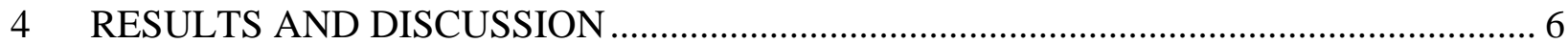

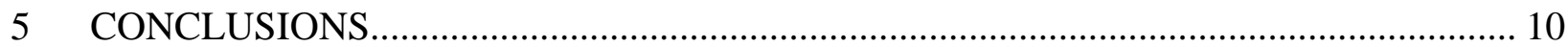

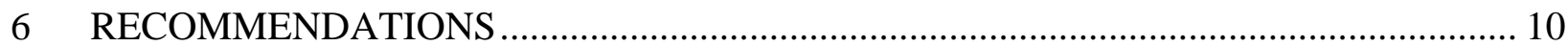

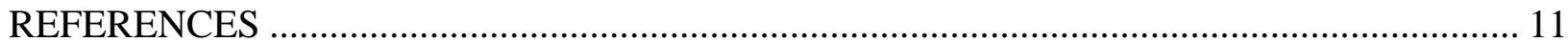

LIST OF SYMBOLS, ABBREVIATIONS, AND ACRONYMS........................................... 12 


\section{LIST OF FIGURES}

Figure 1. The Spectrum of Interest to a Wideband Cognitive Radio Divided into a Set of $N_{b}$ Subbands... 3

Figure 2. Original Sub-band Signal and Compressive Sampling Reconstruction of the Signal in

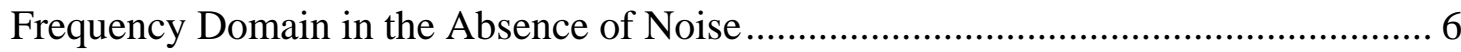

Figure 3. Power Spectral Densities (PSD) of Original and Recovered Signals by Robust Compressive Sampling (CS) with Varying Compression Ratios 33\%, 50\%, 67\%, 84\%

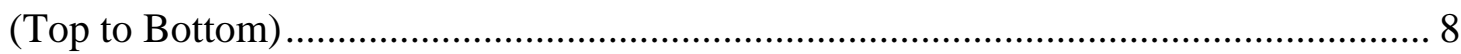

Figure 4. Power Spectra of Original Signal and Those Recovered by the Robust Compressive Sampling Algorithm (With 89 Samples) and by the Periodogram (With 128 Samples) 9 


\section{ACKNOWLEDGMENTS}

This material is based on research sponsored by Air Force Research Laboratory under agreement number FA9453-14-1-0231. The U.S. Government is authorized to reproduce and distribute reprints for Governmental purposes notwithstanding any copyright notation thereon.

\section{DISCLAIMER}

The views and conclusions contained herein are those of the authors and should not be interpreted as necessarily representing the official policies or endorsements, either expressed or implied, of Air Force Research Laboratory or the U.S. Government. 
(This page intentionally left blank)

Approved for public release; distribution is unlimited. 


\section{SUMMARY}

Compressive-sampling based, robust spectrum sensing approach for wideband cognitive radios have been developed. This project was motivated by the fact that wideband cognitive radios can be subjected to heterogeneous spectral activities from various emission sources rendering the resultant noise non-Gaussian. While conventional detectors (e.g., those that are based on least-squares estimates) can be effective against Gaussian noise, they, however, are known to be sensitive to such non-Gaussian nature of noise. Robust detection principles are used to obtain a new spectrum sensing approach that helps alleviate this sensitivity. Moreover, a limiting factor in wideband spectrum sensing is the need for higher sampling rates as necessitated by the Shannon sampling theory. In this project, a compressive sampling front-end is proposed to overcome this hardware imposed limitations on wideband spectrum sensing. Thus, the proposed approach combines a Huber cost function with an $L_{1}$-norm constraint. The proposed compressive sampling based robust detector is indeed shown to give better signal activity detection during wideband spectrum sensing in non-Gaussian noise environments. In particular, the proposed robust method with a smaller number of samples outperforms the conventional periodogram approach in the presence of non-Gaussian noise.

\section{INTRODUCTION}

Cognitive radios present a potential future technology to realize autonomous radio communications over non-contiguous wide spectrum bands in the presence of adverse conditions. These adverse conditions may be both deliberate as well as inadvertent. Moreover, encroachment on previously-allocated spectrum resources by the commercial or unlicensed users and broadband-access can only be expected to grow in the coming years. Combination of these traditional as well as evolving spectrum demands requires future telecommunications technologies to be intelligent, self-aware and spectrally agile. Wideband autonomous cognitive radios (WACRs) pursued in this project are radios with these defining characteristics.

Spectrum awareness is the most salient feature of cognitive radios that makes them cognitive, and spectrum sensing is the process of acquiring spectrum awareness [1-3]. In the case of wideband autonomous cognitive radios, spectrum sensing is usually performed over several non-contiguous spectrum bands, each spanning hundreds of mega-Hertz (MHz) to even

Approved for public release; distribution is unlimited. 
on the order of a giga-Hertz $(\mathrm{GHz})$. Due to wide bandwidth and noncontiguous nature of the frequency range of interest, an effective spectrum sensing framework for a WACR thus needs efficient spectrum scanning, accurate signal detection, as well as signal classification and identification [1]. This complete process of wideband spectrum sensing is referred to as spectrum knowledge acquisition.

In our previous work, we proposed efficient spectrum scanning by first dividing the spectrum range of interest into a set of sub-bands, where each sub-band can be much wider than a single channel assumed in narrowband spectrum sensing [1]. Hence, applying real-time Nyquist-rate sampling to detect spectral activities present in a sub-band can require large sampling rates, leading to very high computational complexity demands for associated signal processing. Another potential challenge in wideband spectrum sensing is the possible heterogeneity of electromagnetic interference (EMI) in a sensed sub-band signal. Many of these man-made interferes (e.g., jammers) are known to not satisfy the convenient Gaussian assumption used in classical signal detection approaches [4].

In this project, we make use of compressive sampling in order to reduce the high sampling rate requirements demanded by wideband sensing. When there is a certain amount of sparsity in the signal with respect to some basis, compressive sampling can be an efficient technique for reconstructing a signal that is sparse with respect to some basis (i.e., most of the expansion coefficients of the signal are zero with respect to a certain basis) $[5,6]$. The efficiency afforded by compressive sampling is two-fold. First, a smaller number of samples compared to what is needed with Nyquist sampling will suffice. Second, the reconstruction of the signal from these reduced number of samples can be achieved with an algorithm with low computationally complexity. We note that, in many situations sensed sub-bands will have low spectrum utilization making them sparse with respect to a frequency-domain basis. This justifies the use of compressive sampling for spectral activity detection in a wideband cognitive radio as in [7]. However, most compressive-sampling based signal reconstruction in the presence of noise commonly assumes Gaussian distributed noise. Hence, direct application of usual compressive sampling reconstruction algorithms for wideband spectrum sensing may lack robustness due to possible non-Gaussian behavior of jammers, interference, and other types of electromagnetic 
radiation [1-4]. As a result, we develop a robust spectral activity detection approach based on compressive sampling for wideband spectrum sensing in a cognitive radio.

\section{METHODS, ASSUMPTIONS, AND PROCEDURES}

Let us consider a wide spectrum band of $B \mathrm{~Hz}$ (where $B$ can be in the order of hundreds of $\mathrm{MHz}$ to even a $\mathrm{GHz}$ ) that is first segmented into several sub-bands. Note that each sub-band may contain several channels, possibly corresponding to different communications systems. As illustrated in Figure 1, let us assume that there are $N_{b}$ sub-bands. In general, scanning of these sub-bands spanning several non-contiguous frequency ranges can be achieved using reconfigurable antennas [8].

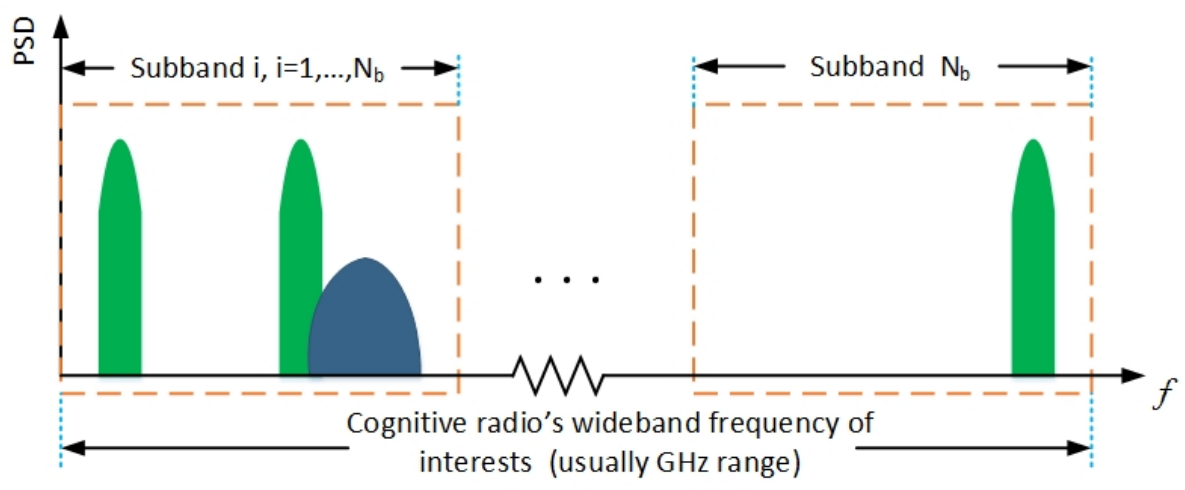

a) at time instant $t=t_{1}$

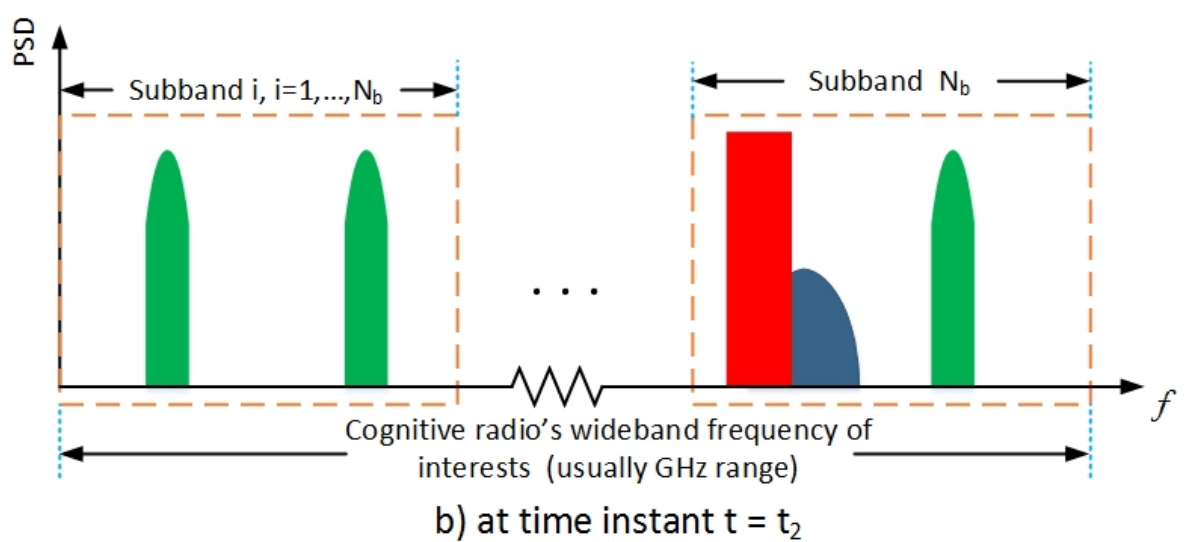

Figure 1. The Spectrum of Interest to a Wideband Cognitive Radio Divided into a Set of $N_{b}$ Sub-bands 
Let us denote the $N$-length discrete-time sub-band signal by $y$, where $N$ is chosen to satisfy the Nyquist sampling criteria. The discrete frequency domain representation, $y_{f}$, of this sub-band signal can then be written as:

$$
\boldsymbol{y}_{f}=\boldsymbol{C} \boldsymbol{y},
$$

where $C$ is a an $N$-point Discrete Fourier Transform (DFT) matrix.

When spectrum utilization within a sub-band is low, as in Figure 1, we may expect $y_{f}$ to be a sparse signal with respect to the frequency domain basis. While conventional ShannonNyquist sampling theory does not take into account such sparsity of signals, compressive sampling allows this sparsity property of a signal to be exploited to detect spectral activities in each sub-band with a reduced number of samples. Indeed, suppose that the sensed signal within the sub-band of interest is sparse and that we only collect an $M$ (where $M<N$ ) number of randomly selected samples from the signal $y$ :

$$
\boldsymbol{y}_{c}=\boldsymbol{\Phi} \boldsymbol{y}=\boldsymbol{\Phi} \boldsymbol{C}^{\boldsymbol{H}} \boldsymbol{y}_{f},
$$

where $\boldsymbol{\Phi}$ is an $M \times N$ random sampling matrix and $y_{c}$ is an $M$-length observation vector. As has been shown in $[5,6]$, if the sampled signal is sparse, then the signal $y$ (or $y_{f}$ ) can indeed be reconstructed from the randomly compressive sampled (under-sampled) version $y_{c}$ of $y$ by solving the following convex optimization problem:

$$
\boldsymbol{y}_{\boldsymbol{f}}^{*}=\arg \min _{\boldsymbol{y}_{f}}\left\|\boldsymbol{y}_{f}\right\|_{l_{1}} \text { subject to } \boldsymbol{y}_{c}=\boldsymbol{\Phi} \boldsymbol{C}^{\boldsymbol{H}} \boldsymbol{y}_{f} .
$$

The lure of compressive sampling lies in the fact that there are algorithms that can solve the above optimization problem efficiently (in terms of accuracy, speed and number of samples) [9, 10]. However, (3) deals with only the ideal noiseless situation. In practice, noise is unavoidable, and thus the compressed sampled signal (2) needs to be modified as:

$$
\boldsymbol{y}_{c}=\boldsymbol{\Phi} \boldsymbol{y}+\boldsymbol{w},
$$

where $w$ is an $M$-length arbitrary noise vector. Most previously proposed compressive sampling algorithms assume that noise, $w$, is Gaussian. As we already discussed, however, this may not often be justifiable in wideband spectrum sensing. Hence, it is desirable to have a wideband spectral activity detector that will be able to withstand possible noise deviations from Gaussian behavior. Thus, a robust compressive sampling approach must be able to handle: 1) a nearly sparse signal, which is justifiable in at least some wideband sensing scenarios, 2) signals effected 
by unknown contaminating noise which is valid for situations in which either the noise is nonGaussian or where there is noise uncertainty (i.e., noise distribution is known only approximately).

A common model for noise with an uncertain distribution is the following $\epsilon$ contaminated distribution $[11,12]$ :

$$
F_{\epsilon}=(1-\epsilon) \Psi+\epsilon H,
$$

where $\Psi$ is a known nominal distribution usually taken to be Gaussian, $H$ is an unknown contaminating distribution that is symmetric and $0<\epsilon<1$ is a known parameter that determines the rate of deviation from nominal Gaussian to non-Gaussian distribution. It is known that for the noise model (5), the detector design problem can be reduced to an optimization involving the following cost function, called the Huber cost function, in place of the usual quadratic cost function encountered in Gaussian noise $[1,11]$ :

$$
l_{H}(x)=\left\{\begin{array}{ll}
x^{2} / 2 & \text { if }|x| \leq \delta_{H} \\
\delta_{H}\left(|x|-\frac{\delta_{H}}{2}\right) & \text { if }|x|>\delta_{H}
\end{array} .\right.
$$

In light of our compressive sampled sub-band sensed signal model (4), in order to obtain a good reconstruction of the possibly nearly sparse signal $y_{f}$ while also combating non-Gaussian noise (5), we thus solve the following optimization problem:

$$
\boldsymbol{y}_{\boldsymbol{f}}^{*}=\arg \min _{\boldsymbol{y}_{f} \in \mathbb{C}^{N}} l_{H}\left(\boldsymbol{y}_{c}-\boldsymbol{A} \boldsymbol{y}_{f}\right)+\gamma\left\|\boldsymbol{y}_{f}\right\|_{l_{1}},
$$

where $\gamma$ is a parameter that can be chosen appropriately to balance between the robustness against noise and the sparsity of the solution. As with (3), there are efficient algorithms that can solve (7).

In this project, we were mainly concerned with comparing the performance of the above compressive-sampling based robust spectrum estimate of the sub-band sensed signal with that of the periodogram, which is optimal if noise were to be Gaussian. However, the performance of interest is two-fold. Both the noise robustness as well as the decrease in number of used samples is of interest. In order to evaluate this performance in a simplified simulation scenario, we considered a sub-band signal $y$ of length $N=128$ composed of three active signals $\left\{x_{1}, x_{2}, x_{3}\right\}$ located at center frequencies (discrete samples per unit time) of 5, 20 and 40, respectively. The corresponding signal amplitudes were arbitrarily chosen to be 8,15 and 22. Each signal has a 
bandwidth of 7 (in discrete frequency) around its center frequency. For random compressive sampling, the sensing matrix $\boldsymbol{\Phi}$ is drawn according to a normal distribution.

\section{RESULTS AND DISCUSSION}

Figure 2 shows the reconstruction of the above sub-band signal with compressive sampling when there is no noise using only $M=102$ number of samples. Note that, as mentioned above, these samples were drawn randomly using a normal distributed sensing matrix. Since, there is no noise, the reconstruction is obtained by solving the standard Basis Pursuit problem of (3) using a primal-dual algorithm [9].
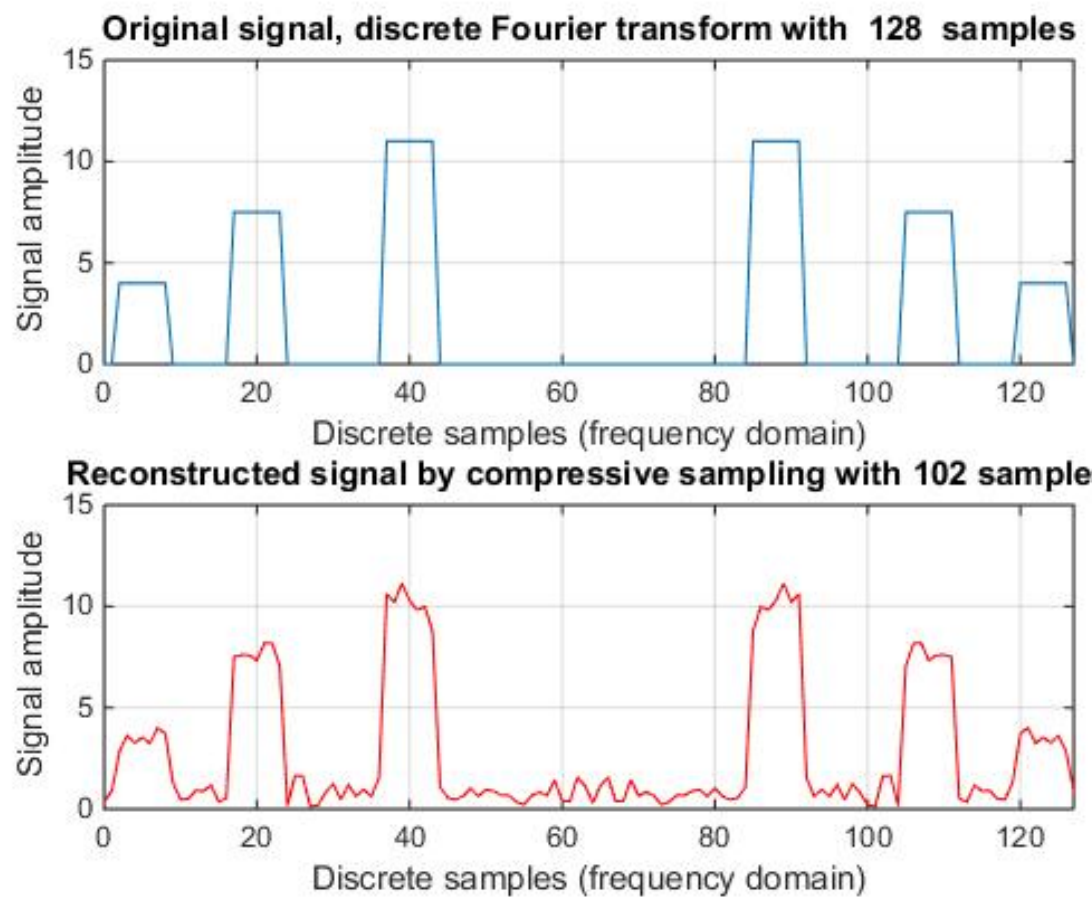

Figure 2. Original Sub-band Signal and Compressive Sampling Reconstruction of the Signal in Frequency Domain in the Absence of Noise 
We may quantify the signal reconstruction performance in terms of either the normalized root mean-squared error (NRMSE) of amplitude spectrum defined as:

$$
N R M S E_{A m p}=\frac{\left\|y_{f}-y_{f}^{*}\right\|_{l_{2}}}{\left\|y_{f}\right\|_{l_{2}}},
$$

or, alternatively, in terms of the normalized root mean-squared error (NRMSE) of power spectrum defined as:

$$
N_{R M S E} E_{P S D}=\frac{\left\|\boldsymbol{y}_{f}{ }^{2}-\boldsymbol{y}_{f}{ }^{2}\right\|_{l_{2}}}{\left\|\boldsymbol{y}_{f}{ }^{2}\right\|_{l_{2}}} .
$$

In the noiseless case of Figure 2, $N R M S E_{A m p}$ is found to be 0.2036 .

Next, we introduce noise in to the above sub-band signal model. In particular, we assume that the signal is corrupted by Gaussian-Laplacian mixture noise with $\epsilon=0.9$. The standard deviation of the zero-mean nominal Gaussian noise is taken to be 1 while the parameter of Laplace noise distribution is set to 0.2 . Figure 3 shows the reconstructed signal by solving (7) as we vary the compression ratio of the number of samples from $33 \%$ to $84 \%$ (with respect to the Nyquist rate). As can be seen from Figure 3, the signal reconstruction performance steadily improves as the number of samples is increased. Importantly, even at the high compression ratio of $67 \%$, the performance of the reconstructed signal seems to be reasonable enough for signal activity detection in the presence of noise. 

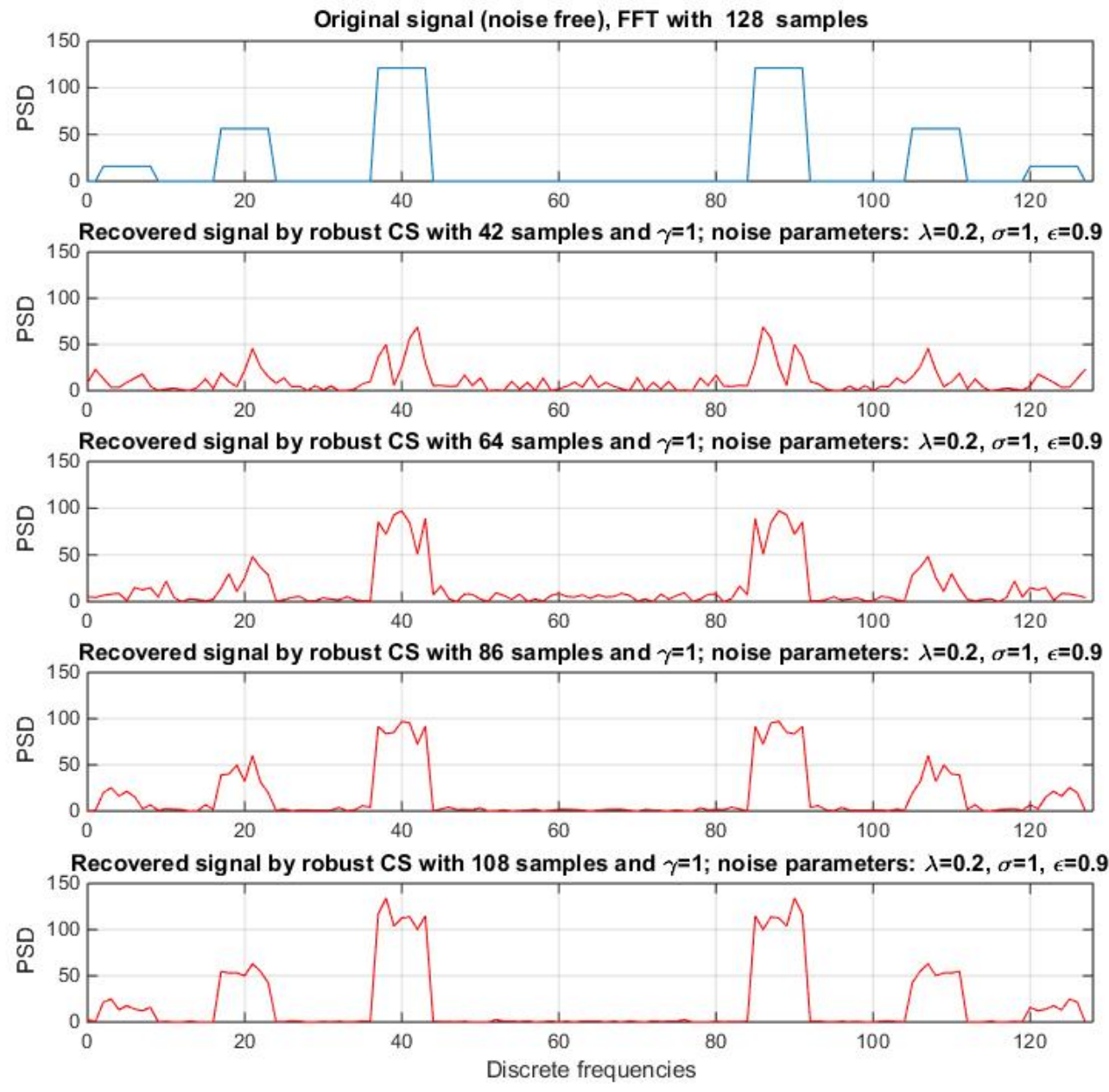

Figure 3. Power Spectral Densities (PSD) of Original and Recovered Signals by Robust Compressive Sampling (CS) with Varying Compression Ratios 33\%, 50\%, 67\%, 84\% (Top to Bottom)

It is worth comparing the signal reconstruction performance of the proposed compressive-sampling based algorithm to that of the standard periodogram. Figure 4 shows the original signal (power) spectrum along with those reconstructed by robust compressive sampling (7) using $M=89$ samples and the periodogram using 128 samples. Note that, the signal is corrupted by the Gaussian-Laplacian noise as assumed above. While still using about 30\% less number of samples compared to the periodogram, the proposed robust compressive sampling 
based algorithm seems to provide somewhat better reconstruction performance than the periodogram. Indeed, we found that the robust compressive sampling with $M=89$ samples gives a spectrum reconstruction with $N R M S E_{A m p}=1.3578$, while periodogram reconstruction has $N R M S E_{A m p}=1.4102$.
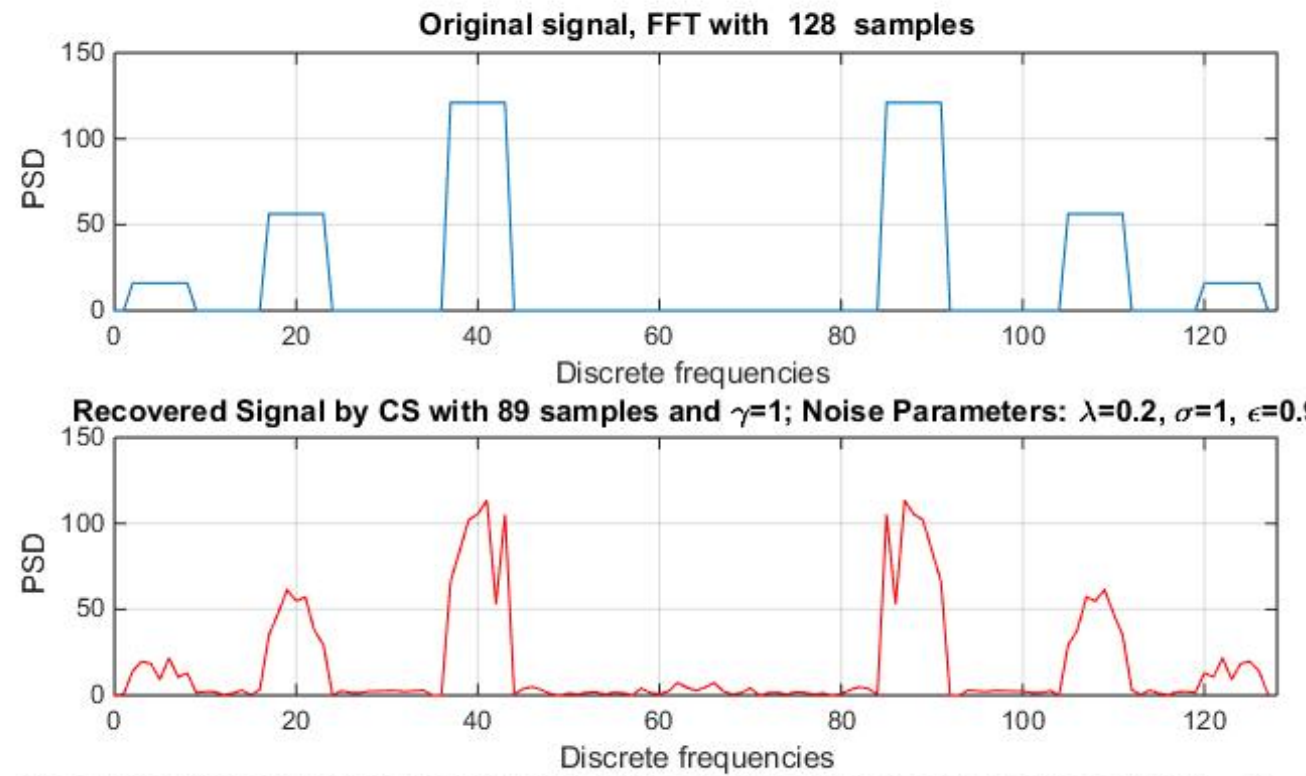

Recovered Signal by Periodogram with 128 samples and Noise Parameters: $\lambda=0.2, \sigma=1, \epsilon=0.9$

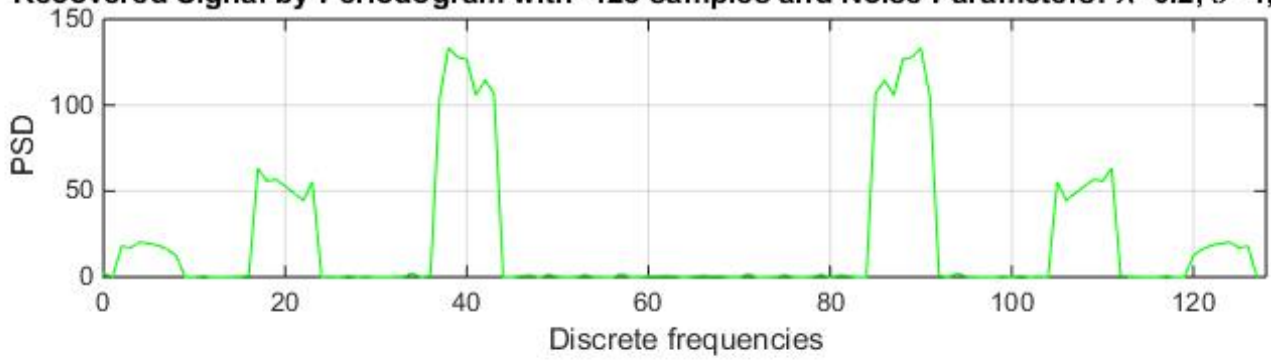

Figure 4. Power Spectra of Original Signal and Those Recovered by the Robust Compressive Sampling Algorithm (With 89 Samples) and by the Periodogram (With 128 Samples) 


\section{CONCLUSIONS}

In this project, we developed a compressive-sampling based, robust wideband spectrum sensing approach for cognitive radios. The proposed method augments the Huber cost function with an additional $L_{1}$-norm penalty term in order to find a sparse spectrum estimate while achieving robustness against possibly non-Gaussian noise. Through simple simulated examples, we observed that the proposed approach can improve the wideband spectrum sensing performance in two important ways: 1) the required number of samples can be reduced, and 2) the estimation performance can be better than that of the conventional periodogram. This allows us to possibly replace the usual conventional Nyquist-rate based front-end with a compressivesampling based front-end that will facilitate real-time sensing of wide spectrum bands in the presence of possibly non-Gaussian man-made interference and other EMI.

\section{RECOMMENDATIONS}

The compressive-sampling based robust wideband spectrum sensing approach developed in this project is just a one component in a wideband autonomous cognitive radio system. The immediate next-step of this work is to test this algorithm on various realistic channel and spectrum conditions and refine it for satisfactory performance in real-time. The longer-term objective is to develop the other modules of the wideband autonomous cognitive radio system and finally develop an integrated complete system. Thus, it is recommended that the proposed algorithm be refined for more realistic spectrum sensing environments by incorporating machine learning techniques and then be integrated with other modules of a cognitive radio front-end processing.

Approved for public release; distribution is unlimited. 


\section{REFERENCES}

1. S. K. Jayaweera, Signal Processing for Cognitive Radios, 1st ed., New York, NY, USA, John Wiley \& Sons Inc., 2014.

2. J. Mitola and G. Q. Maguire Jr, “Cognitive Radio: Making Software Radios More Personal,” IEEE Personal Communications, Vol. 6, No. 4, pp. 13-18, 1999.

3. S. Haykin, "Cognitive Radio: Brain-Empowered Wireless Communications," IEEE Journal on Selected Areas in Communications, Vol. 23, No. 2, pp. 201-220, 2005.

4. M. Bkassiny and S. K. Jayaweera, "Robust, non-Gaussian Wideband Spectrum Sensing in Cognitive Radios," IEEE Transactions on Wireless Communications, Vol. 13, No. 11, pp. 6410-6421, 2014.

5. D. L. Donoho, “Compressed Sensing," IEEE Transactions on Information Theory, Vol. 52, No. 4, pp. 1289-1306, 2006.

6. E. J. Candes and M. B. Wakin, “An Introduction to Compressive Sampling,” IEEE Signal Processing Magazine, Vol. 25, No. 2, pp. 21-30, 2008.

7. Z. Tian and G. B. Giannakis, "Compressed Sensing for Wideband Cognitive Radios," in Proc. IEEE Int. Conf. Acoust., Speech, Signal Process. (ICASSP), Honolulu, HI, Apr. 2007, pp. IV-1357-IV-1360.

8. Y. Tawk, S. K. Jayaweera, C. Christodoulou, and J. Costantine, “A Comparison Between Different Cognitive Radio Antenna Systems," in Proc. International Symposium on Intelligent Signal Processing and Communication Systems (ISPACS), Chiangmai, Thailand, pp. 1-6, Dec. 2011.

9. $\quad$ E. Candes and J. Romberg, " $\mathrm{L}_{1}$-magic: Recovery of Sparse Signals via Convex Programming,” URL: www. acm. caltech.edu/l1magic/downloads/l1magic. pdf, Vol. 4, 2005.

10. A. Y. Yang, S. S. Sastry, A. Ganesh, and Y. Ma, "Fast $\mathrm{L}_{1}$-minimization Algorithms and an Application in Robust Face Recognition: A Review," in Proc. IEEE Int. Conf. on Image Process. (ICIP), Hong Kong, pp. 1849-1852, 2010.

11. P. J. Huber and E. M. Ronchetti, Robust Statistics, $2^{\text {nd }}$ ed. New York, NY, USA, John Wiley \& Sons Inc., 2009.

12. H. V. Poor, An Introduction to Signal Detection and Estimation, $2^{\text {nd }}$ ed. New York, NY, USA, Springer-Verlag, 1998. 
LIST OF SYMBOLS, ABBREVIATIONS, AND ACRONYMS

\begin{tabular}{|l|l|}
\hline$B$ & Frequency Band \\
\hline CS & Compressive Sampling \\
\hline DFT & Discrete Fourier Transform \\
\hline EMI & Electro Magnetic Interference \\
\hline FFT & Fast Fourier Transform \\
\hline GHz & Giga Hertz \\
\hline $\mathrm{Hz}$ & Hertz \\
\hline $\mathrm{L}_{1}-$ norm & Vector measurement \\
\hline MHz & Mega Hertz \\
\hline$N_{b}$ & Number of sub-bands \\
\hline NRMSE & Normalized Root Mean-Squared Error \\
\hline PSD & Power Spectral Density \\
\hline WACR & Wideband Autonomous Cognitive Radio \\
\hline
\end{tabular}

Approved for public release; distribution is unlimited. 


\section{DISTRIBUTION LIST}

DTIC/OCP

8725 John J. Kingman Rd, Suite 0944

Ft Belvoir, VA 22060-6218

1 cy

AFRL/RVIL

Kirtland AFB, NM 87117-5776

2 cys

Official Record Copy

AFRL/RVSV/Steven A. Lane 1 cy

Approved for public release; distribution is unlimited. 
(This page intentionally left blank)

Approved for public release; distribution is unlimited. 